\title{
Comprendre les transidentités
}

Par Arnaud Alessandrin (sociologue, université de Bordeaux)

Remerciements à Anne-Sophie Le Toullecq (professeure des écoles)

«C'est à ma façon

Si je ne veux pas être une grande fille

Je serai un petit garçon

Pour chaque insulte lancée

Il pousse un grain de beauté »

(Christine and the queens, « Half Ladies », album Chaleur humaine, 2014)

Depuis quelques années les mouvements LGBTQI - lesbiennes, gays, bisexuels, transgenres, queers et intersexes - gagnent en visibilité. Les transgenres ont récemment conquis des droits (tout particulièrement en France). Mais ils se heurtent toujours aux normes de genre, qui s'abattent sous des formes variées mais violentes : discriminations transphobie, psychiatrisation des parcours de santé, maintien de stéréotypes et de préjugés innombrables. Devenir trans et vivre trans est-il devenu plus facile ou, tout au moins, maintenant possible ?

\section{Qu'entend-on par « transidentités »?}

Répondre à cette question suppose un léger saut dans le temps. En 1953, l'endocrinologue Harry Benjamin fait adopter l'idée que les personnes qu'il nomme «transsexuelles » sont atteintes d'un trouble, distinct de l'homosexualité, qui s'enracine dès le plus jeune âge et qui se manifeste par un désir incompressible de changer de sexe. Cette permanence de la demande, associée à la souffrance qui doit en découler, fonde la première définition du «transsexualisme». Avec la conceptualisation de Harry Benjamin, les 
personnes «transsexuelles » passent du statut d'anormales à celui de personnes « malades ». Cette catégorisation fait aujourd'hui bondir, à juste titre. Mais ce glissement promet à l'époque une prise en charge des demandes des patientes et des patients. Pour autant, penser qu'il y a toujours eu des personnes «transsexuelles » relève d'un anachronisme conceptuel. La vie de Lili Elbe, une des premières personnes à subir une opération de réassignation dans les années 1930, en est un témoignage flagrant. En effet, lorsque Lili Elbe est admise à l'hôpital pour son opération (elle en subira en réalité plusieurs, notamment-une greffe d'utérus dont elle décédera), elle est admise en tant que «patient homosexuel » demandant une «stérilisation ». C'est dire combien nos «visions des autres », leurs définitions, leurs « catégorisations » forgent non seulement les représentations de ce qu'il faut leur faire, mais également nos actions, en matière de santé et de prise en charge notamment.

Mais revenons au concept de «transsexualisme ». Il se sédimente vite après son acceptation clinique. En France, dès 1956, on recense des thèses sur la question. La décennie qui suit est de nouveau marquée par une effervescence scientifique autour de ce thème, mais la France se démarque d'autres pays, comme les États-Unis par exemple, par la présence excessivement forte des discours psychanalytiques qui, ailleurs, sont progressivement remplacés par des études psychiatriques, endocrinologues et chirurgicales pour décrire le «transsexualisme ».

Cette spécificité française aura, jusqu'à aujourd'hui, des conséquences sur la manière de prendre en charge les personnes trans. Si nous traversons l'Atlantique, toujours dans les années 1960, les thèses autour du concept de «genre » se multiplient. Le cas d'Agnès, décrit par le sociologue Garfinkel, illustre le passage progressif de la question médicale du «transsexualisme» à la question «sociale». Il ne s'agit plus de savoir seulement « pourquoi » les personnes «transsexuelles » expriment un désir de changer de sexe, mais davantage de savoir « comment » elles y parviennent, à l'encontre des normes et de traditions puissantes. C'est dans cet esprit que des gender clinic verront le jour aux États-Unis, afin de répondre aux demandes des personnes concernées.

Un point de vocabulaire important : en réalité les personnes ne «changent » pas de genre. Elles deviennent qui elles sont !

La France reste en retrait de ces débats. La question du genre, hier comme aujourd'hui, est mal perçue dans notre pays. Il faut attendre le tout début des années 1980, et 
1981 pour être précis, pour que la Sécurité sociale entérine le premier protocole de changement de sexe (Paris, Lyon, Bordeaux et Marseille en bénéficieront). Avant 1981, l'opération de changement de sexe est considérée par l'Assurance maladie et l'Ordre des médecins comme une mutilation. Dans ce contexte, les opérations ont souvent lieu à l'étranger, notamment dans la très controversée clinique du professeur Burou à Casablanca.

Après 1981, des protocoles sont donc instaurés. Le suivi protocolaire est fixé de la sorte : la psychiatrie diagnostique le «transsexualisme » de la personne, avant de l'envoyer vers un endocrinologue puis un chirurgien. Notons à cet égard qu'il n'y a pas, véritablement, de diagnostic du «transsexualisme ». Il s'agit d'un diagnostic différentiel qui vise à écarter les demandes qui en prendraient la forme sans en être, comme les troubles schizophrènes. Dans les écrits des équipes protocolaires, cette étape psychiatrique vise à délimiter une frontière entre de «vrais » et de «faux » patients. Elle vise aussi à limiter et éviter ce qui relèverait du « regret », à savoir les personnes qui désireraient une nouvelle fois changer de sexe après l'opération. Une revue de la littérature quelque peu sérieuse nous permet de constater que, en moyenne, $1 \%$ des personnes prises en charge émettent des regrets postopératoires. Toutefois, à la lecture de ces « regrets », il convient d'observer que ces derniers ne renvoient pas au changement en tant que tel, mais plus à la technique opératoire, ou bien encore au suivi médical lui-même. Mais, au total, les critères de prise en charge restent à ce point stricts que le nombre de personnes inscrites en file active auprès d'endocrinologues ou de psychiatres hospitaliers est démesurément supérieur au nombre de personnes opérées. Pour reprendre les termes du chercheur Alain Giami, l'offre de soins ne répond aucunement à la demande.

\section{Qu'est-ce qui a changé dans la prise en compte des transidentités ?}

Depuis les années 1990 est à l'œuvre un triple phénomène, un triple débordement des « catégorisations » du «transsexualisme » et des façons de l'appréhender. Tout d'abord, les

personnes trans finissent par se rassembler. À l'image des personnes homosexuelles, la structuration associative et communautaire permet une déprise de l'isolement, de la honte, du tabou. Elle autorise également des échanges d'expériences, des comparaisons entre les 
parcours, entre les prises en charges, et favorise une entre-aide bienvenue. Assez rapidement, Internet aidant, les associations trans se multiplient et se transforment, jusqu'à devenir des espaces de contre-expertises face aux discours médicaux et des interfaces politiques, autour des notions de genre ou de lutte contre la transphobie, par exemple

Deux autres débordements accompagnent cela. Le premier renvoie aux avancées juridiques. Après de multiples condamnations par la Cour européenne de justice, la France octroie aux personnes trans des droits qui jusque-là leur étaient refusés. Avant 2016, la transphobie n'était pas, en tant que telle, reconnue dans le droit français. Toujours avant 2016, les personnes trans devaient fournir la preuve d'une «irréversibilité » de l'opération pour obtenir enfin un nouvel état civil. Cette notion d'irréversibilité était entendue par les tribunaux comme une stérilisation. Cela signifie qu'un homme trans ne pouvait garder son utérus et, inversement, qu'une femme trans ne pouvait garder son pénis. Plus encore, les gamètes des personnes n'étaient pas préservés avant l'opération (et ne le sont d'ailleurs pas toujours), alors même que, dans des cas similaires, comme lors d'un cancer des testicules, les gamètes des patients sont conservés. Cette exigence de stérilisation rendait, à proprement parler, la vie des personnes trans invivables, car que fait-on sans un état civil qui correspond à notre genre? Vivre le «transsexualisme» suppose toujours de lutter contre les discriminations, et les équipes hospitalières réunies autour de la Société française d'étude et de prise en charge du «transsexualisme » (SOFECT) sont régulièrement prises pour cible.

Un troisième débordement doit enfin être souligné, un débordement sémantique. Avec l'essor de la théorie «queer» et des mouvements liés aux minorités sexuelles et de genre, les identités de genre et de sexualité se sont mises à fourmiller. L'hétérosexualité et la « cisidentité » (le fait de ne pas vouloir changer de genre) ne sont plus des modèles normatifs aussi forts. Ou, plutôt, ils tendent progressivement à s'effriter. Lentement mais assez inéluctablement. Ainsi, en 2015, nous établissions la première mesure statistique de la transphobie en France, et que nous demandions aux personnes concernées «quel est votre genre? », nous avions obtenu près de 36 réponses différentes. Hommes, femmes, trans, hommes trans, «transsexuels », bigenres, agenres... Cette explosion des identités signale un éloignement brutal entre les assignations binaires (femme-homme), les expressions et les expériences de genre. Plus encore, en 2018, lorsque la recherche «santé LGBTI» a été rendue publique, 42 identités de genre différentes ont été déclarées à la question «quel est 
votre genre ?». C'est dire combien, pour les personnes trans, le modèle «transsexuel» d'hommes « qui deviennent » des femmes, et inversement, constitue un modèle obsolète, qui ne répond que partiellement aux demandes de prise en compte contemporaines. L'explosion des termes comme «non binaires » et l'émergence de figures médiatiques se revendiquant comme tel parachèvent ce phénomène.

\section{«Surtout, je suis moi »}

«J'ai du mal à me retrouver dans cette “identité" de trans qu'on me colle, je suis trans c'est vrai, j'assume, je revendique même, et en même temps je suis une femme, une femme parmi les autres, voilà, c'est tout. Surtout, je suis moi, enfin moi, je dirais. J'ai eu un corps de garçon, mais je ne suis pas un garçon, je ne me sens pas être un garçon, jamais je ne me suis sentie être un garçon. Petite, j'ai eu vraiment honte de mon sexe, même avant de savoir ce que c'était vraiment. Je pensais qu'il y avait quelque chose d'anormal entre mes jambes, une difformité. J'avais peur qu'on découvre que j'étais une petite fille différente, même vers cinq, six ans, je ne voulais pas me déshabiller devant quelqu'un.

Ensuite, à l'école, dans la cour, j'ai tout de suite eu l'air bizarre : beaucoup trop calme, beaucoup trop réservée pour un "garçon", et "pourquoi est-ce que tu ne vas pas jouer au foot avec tes copains?". Je finissais par me mettre dans un coin, avec un livre, en regardant avec envie les filles, au loin, qui discutaient entre elles. Je me suis longtemps sentie très seule.

J'ai une sœur, et mon père est gendarme, comme son père et mon arrière-grand-père avant lui. Il a fondé beaucoup d'espoir en moi. Petite, je pouvais faire des trucs "de fille" avec ma sœur, sans éveiller les soupçons. J'avais une dînette, des poupées, je passais mon temps à jouer avec les autres filles. Mais, en grandissant, mes parents ne comprenaient pas ce qui se passait, ça me rendait infiniment triste...

Lorsque j'ai atteint la puberté, c'est devenu l'enfer. Les érections, c'était difficile à supporter, à sentir ce corps dans lequel je ne me reconnaissais pas, qui s'imposait à moi. Ce corps me dégoûtait, et cette voix, cette voix qui changeait elle aussi, malgré moi, je ne pouvais plus parler sans avoir honte. Je chuchotais quand il fallait absolument s'exprimer.

À force de moqueries et de souffrances au collège, j’ai fini par essayer de ressembler 
à un garçon, j'ai tenté de m’intéresser aux filles ; ça n'a pas marché. Au lycée, après le sport, dans les douches communes, mon désir amoureux pour les garçons s'est transformé en désir sexuel.

Impossible de faire autrement, impossible de sentir les choses autrement, malgré tous mes efforts pour me masculiniser à outrance, j'ai commencé à penser que j'avais une maladie mentale, je ne connaissais personne qui soit LGBT.

À l'époque, Internet a été une vraie bouffée d'oxygène : je pouvais choisir qui j'étais, comment je m'appelais. Au début, j'ai créé des profils de garçon mais très vite je me suis sentie vraiment plus heureuse avec des profils féminins. C'est avec un de ces profils que j'ai rencontré ma première copine. Cette rencontre, je ne l'oublierai pas, car c'est elle qui m'a vraiment fait prendre conscience des choses, qui m'a poussée, avec beaucoup d'affection et de tact, à accepter pleinement les choses et qui j'étais, ce que je ressentais. Elle m'a accompagnée à ma première réunion avec une association avec des personnes trans. C'est là que j'ai compris, même si c'est un peu bizarre de dire ça comme ça. En tout cas, j'ai compris beaucoup de choses. C'est à ce moment-là que j'ai pris une décision : je commencerai une transition après la fac, après mes études. D'ici-là, il faut faire avec, faire avec ce corps, en attendant de vivre dans la peau d'une fille, de se sentir vraiment comme je suis, être pour de bon Élisa, ou Lisa, pour mes amis proches. »

Au total, le «transsexualisme » est peu à peu remplacé par des façons d'être soi, dans son sexe, comme dans son genre, que le terme parapluie «transidentités » ou «personnes trans» traduit avec plus d'inclusion et moins d'épaisseur pathologisante. D'ailleurs, les nomenclatures comme le DSM (Diagnostic et Statistique des Troubles Mentaux) épousent ce glissement vers une reconnaissance de genre plus fluide et une pathologisation moindre.

\section{Les luttes transidentitaires}

Où en sont les luttes transidentitaires aujourd'hui ? Dans un contexte de transphobie dure (une enquête de 2019 relève que $98 \%$ des personnes trans ont vécu de la transphobie dans l'espace public, et que $38 \%$ des témoins d'agression ont fini par prendre part aux 
violences $^{1}$ ), quelles sont les revendications portées par les personnes concernées? Que pouvons-nous dire de l'actualité des questions trans?

Premièrement, il s'engage aujourd'hui une véritable reconnaissance de la diversité des formes de transidentités. Par exemple, les questions de fluidité de genre se posent avec une acuité toute particulière. Qu'entend-on par fluidité de genre ? C'est principalement l'idée selon laquelle le genre assigné à la naissance ne se reproduit pas en une identité de genre fixe et définitive chez l'individu (homme, femme, masculin, féminin). Cela signifie aussi que l'identité de genre n'est, chez certaines et certains, ni centrale ni décisive pour se définir. Pour d'autres, dans un geste plus politique, se défaire des identités de genre, c'est se défaire des normes vécues comme oppressantes. Cela peut paraitre très théorique. Toutefois, les récentes recherches tendent à montrer l'importance numérique de ce phénomène, notamment chez les plus jeunes. Or, les institutions, scolaires, juridiques ou médicales, ne semblent pas à même de répondre convenablement à ces nouvelles demandes.

Deuxièmement, poursuivre les efforts associatifs, juridiques et politiques en termes de meilleure défense des personnes transidentitaires reste au centre des préoccupations. Les notions d'accès aux droits, de démocratie sanitaire et de lutte contre les discriminations se superposent ici, et le lien entre santé et société est à cet endroit particulièrement fort : défaire les transitions de la pathologie, de l'anormalité, c'est éloigner encore un peu plus les personnes trans de la transphobie. Différentes voies sont entrouvertes, notamment l'assouplissement des parcours protocolaires en vue d'une prise en charge des parcours non hospitaliers.

Enfin, un troisième mouvement, encore timide, doit être initié : celui qui consiste à mieux prendre en charge la santé globale des personnes trans. Qu'en est-il aujourd'hui de la qualité des opérations génitales? Si elles ont nettement progressé du point de vue technique, la réputation de ces opérations ne s'est, quant à elle, pas beaucoup améliorée. Qu'en est-il de la formation des endocrinologues en matière d'hormonothérapie et, notamment, des risques cancérigènes liés à ces hormones ? Qu'en est-il de l'accès aux bloquants hormonaux pour les mineurs trans ? Qu'en est-il du droit à la parentalité et à la reproduction des personnes trans, bref, qu'en est-il de la préservation des gamètes des personnes opérées? Enfin, qu'en est-il

1. Arnaud Alessandrin, Johanna Dagorn et Chad Charasse, "Ville et LGBTI-phobies », rapport de l'Observatoire bordelais de l'égalité, 2019. 
de l'accès aux opérations? La psychiatrie doit-elle toujours être la porte d'entrée des protocoles hospitaliers vers le changement de sexe? Autant de questions qui, malheureusement, forcent à ne pas penser la prise en compte actuelle des transidentités comme étant acquise.

\section{Morale de l'histoire}

A grands coups de psychiatrisation, de stéréotypes et de préjugés discriminants, les transidentités ont été poussées du côté de l'abjecte, du secret, du dégout, parfois même du dégout de soi. Depuis quelques décennies nous assistons au mouvement inverse. La fierté, la visibilité, la parole des personnes concernées : tout concorde vers une déprise vis-à-vis de la pathologie et de la stigmatisation. Pourtant, des résistances persistent. C'est bien que le genre ne se laisse pas si facilement défaire. Peut-être que, pour éviter une glaciation des positions entre une société qui freine et des minorités qui avancent sans elle, ils convient de toujours être pédagogue, de laisser une place, aux personnes comme aux nouveaux mots, aux nouvelles identités, afin de ne jamais laisser se sédimenter des normes excluantes, et ce dès les plus jeunes âges.

\section{Lexique de désenfumage}

Cisidentité : sont nommées « cisgenres » ou «cisidentitaires » les personnes dont le sexe de naissance correspond, sinon parfaitement, du moins assez grandement à l'identité de genre de la personne. Les personnes cisgenres sont à cet égard des «non-trans». La cisidentité permet de rappeler que les minorités ne sont pas seules à être «particulières » et, par conséquent, que les majorités (ou ceux qui sont jugés comme «normaux ») sont aussi une composante «particulière» de la société, qu'il convient de nommer. Si le couple «trans/cis » s'est mutuellement créé, seule la cisidentité est pleinement restée du côté du normal et du normatif.

Mineurs trans: encore aujourd'hui, la transidentité renvoie (dans les imaginaires collectifs) à une figure adulte. Or, il existe des mineurs qui expriment des identités de genre non conformes au genre assigné à la naissance. Afin de ne pas limiter ces expressions à des 
"phénomènes de mode», les associations et les chercheurs anglo-saxons ont inventé le terme de gender creativ kids ou d'enfants trans. Si ceci ne dit pas que toutes les expressions de genre dissidentes en deçà de dix-huit ans donneront lieu à des parcours trans, cela souligne qu'il existe potentiellement des personnes trans même chez les mineurs.

Genre : le «genre est un arsenal catégoriel qui classe», «en ce que les valeurs portées par le pôle masculin sont considérées comme supérieures à celles portées par l'autre pôle», selon l'anthropologue Françoise Héritier. La chercheuse Marie-Joseph Bertini complète : «Le genre (ce que l'on entend par féminin et masculin) peut être défini comme l'ensemble construit des rôles et des responsabilités sociales assignées aux femmes et aux hommes à l'intérieur d'une culture donnée à un moment précis de son histoire ${ }^{2}$.» Cette socialisation différenciée et hiérarchisante nous donne des indications sur ce que n'est donc pas, en creux, le genre, c'est-à-dire ni une donnée purement biologique, ni une évidence historique, ni même une norme fixe (socialement comme individuellement). Toutefois, un certain nombre de normes de genre ne cessent de se répéter (avec plus ou moins de succès dans l'histoire et des individus ciblés) : l'idée d'une différence fixe entre des catégories (femme/homme), l'idée d'une naturalité des catégories, l'idée d'une inégalité justifiée entre les catégories (ce que l'on nomme le patriarcat) et l'hétérosexualité. Lorsque ces normes ne sont pas respectées, il s'abat bien souvent sur les individus une «police de genre », c'est-àdire des sanctions face à la transgression ${ }^{3}$. Il existe donc à cet égard des inégalités et à des discriminations genrées que l'on retrouve dans les carrières des femmes, dans le partage des tâches ménagères, dans les orientations scolaires ou dans les rôles publics incarnés par les femmes comme par les hommes. On dit bien d'ailleurs que le masculin l'emporte sur le féminin! Enfin, le genre est aussi relatif à la notion d'identité. C'est d'ailleurs ce que l'on nomme l'«identité de genre ». Si les comportements inhérents au genre font l'objet d'un long apprentissage, c'est aussi qu'ils sont susceptibles d'évoluer. Dans sa célèbre phrase, la philosophe américaine Judith Butler résume cela de la sorte : le genre est « une pratique d'improvisation qui se déploie à l'intérieur d'une scène de contrainte ${ }^{4}$ ».

2. Marie Joseph Bertini, Ni d'Éve ni d'Adam, Max Milo, Paris, 2006.

3. Esteve-Bellebeau Brigitte et Alessandrin Arnaud, Genre! L'essentiel pour comprendre, Des ailes sur un tracteur, 2014.

4. Judith Butler, Défaire le genre, Amsterdam, 1999. 
Intersexe: se dit de personnes nées avec des caractéristiques sexuelles qui ne correspondent pas à la différenciation binaire masculin/féminin, pour lesquelles la médecine est intervenue soit chirurgicalement, soit (et) par le biais d'hormones afin de normaliser l'appareil génital. La médecine procède alors dès le plus jeune âge à des interventions médicales non consenties afin d'assigner un sexe à l'enfant. Les personnes intersexes sont ainsi marquées par de nombreuses interventions qui ne prennent pas en compte leur identité de genre. Aujourd'hui, si de nombreux pays réclament l'arrêt de ses opérations ou y sont parvenus, la France poursuit son programme d'assignation d'un sexe à la naissance.

Queer: le terme est un mot anglais qui renvoie à une insulte (étrange, tordu ou malade). Mais, dans les années 1990, en procédant par renversement du stigmate, des groupes LGBT se réapproprient le terme afin d'en phagocyter la charge péjorative. «Queer» devient alors une identité. Mais le queer est aussi un mouvement, en provenance des marges sexuelles de la société, qui défend le nomadisme de l'identité (son aspect non essentiel). Le mouvement queer est aussi une critique des tentatives de normalisations et d'assimilation des marges aux normes coercitives. Le mouvement queer épouse également la forme de productions théoriques et la «théorie queer » à son tour va fermement critiquer les dispositifs normatifs. S'il s'agit là d'une théorie générale critique, c'est également une pratique au sens ou le mouvement queer encourage la performance et la création comme outil de déconstruction des normes.

Transgenre : se dit de personnes dont le genre et/ou le sexe ne correspond pas, partiellement ou complètement, au sexe assigné à la naissance. Différentes transitions sont alors possibles. On entend souvent parler de personne «transsexuelle » mais ce terme à connotation pathologisante ne sera pas retenu dans le cadre de cet article qui traite, entre autres, de maltraitance nominale. Le «transsexualisme » renvoie en effet à une nosographie (description médicale) psychiatrique, alors que les parcours transidentitaires ne sont pas réductibles à l'idée d'un trouble ou d'une maladie. Bien au contraire, ils participent plutôt d'une nouvelle approche des parcours de vie et des parcours de genre, moins statique. On emploiera de manière indistincte le terme de «transgenre » et de «trans », terme parapluie qui englobe les personnes trans, c'est-à-dire les personnes dont le sexe assigné à la naissance ne correspond pas à l'identité de genre vécue. 
Transphobie: la transphobie est une discrimination à l'encontre des personnes transidentitaires. Reconnue depuis juillet 2012 dans le droit français, la transphobie recouvre différentes réalités. Nous distinguerons une transphobie «directe», découlant immédiatement d'un rejet de la transidentité, et une transphobie «indirecte» mettant en scène des allants de soi cisgenres en défaveur des trans (sans pour autant que la transidentité soit connue). Ces deux figures de la transphobie pourraient être nommées « relationnelles » et portent sur l'ensemble des mots et des représentations quotidiennes dans un monde cisgenré (non trans). Mais il faudrait souligner un autre type de transphobie, « institutionnelle » celle-ci, et qui, en limitant l'accès au changement d'état civil et aux opérations remboursées, rend vulnérable l'ensemble des personnes souhaitant faire une transition selon leurs propres modes de transition (chirurgicale, hormonale, nominale, etc.). Il faudrait ajouter à cela deux dimensions centrales dans la production de la transphobie : la «maltraitance théorique », c'est-à-dire la transphobie inscrite dans les définitions et articles scientifiques, ainsi que la «maltraitance médiatique» qui solidifie des représentations stéréotypées des personnes trans.

Transsexuel : le «transsexualisme » est défini comme une maladie mentale dés. Ce terme est donc très fortement connoté et tend à pathologiser les personnes ainsi qualifiées. Le concept de «transsexualisme », connu aussi sous le vocabulaire plus récent de «dysphorie de genre », se caractérise par un dégoût de son sexe et une demande persistante à en changer. C'est cette définition stricte qui sera adoptée dans de nombreux pays - dont la France - pour baliser des parcours de soins. Très critiqué par les associations et les personnes concernées, ce terme induit une maltraitance dans les mots mais aussi dans les pratiques médicales.

\section{$\underline{\grave{A} \text { lire }}$}

Ali Aguado et Ian Zdanowicz, «L'usage du droit dans le mouvement d'émancipation trans », Cahiers du genre, vol. 57, n 2, 2014, p. 77-94

Arnaud Alessandrin, Actualité des trans studies, AEC, 2019

Arnaud Alessandrin, Sociologie des transidentites, Le Cavalier Bleu, Paris, 2018

Arnaud Alessandrin et Johanna Dagorn, «La santé des LGBTI à l'école », L'École des parents, $\mathrm{n}^{\circ} 627,2018$, p. $28-29$ 
Arnaud Alessandrin, « Pour en finir avec la notion de troisième sexe », Socio, $\mathrm{n}^{\circ} 8$, novembre 2017

Arnaud Alessandrin et Anastasia Meidani, «Cancers et transidentités : une nouvelle population à risques ? », Sciences sociales et santé, vol. 35, n 1, 2017, p. 41-63

Arnaud Alessandrin, « La transphobie en France : insuffisance du droit et expériences de discrimination », Cahiers du Genre, vol. 60, no. 1, 2016, pp. 193-212.

Arnaud Alessandrin, «Ce que le DSM V fait aux variances de genre », Socio-Logos, $\mathrm{n}^{\circ} 9,2014$

Arnaud Alessandrin et Brigitte Bellebeau, Genre! L'essentiel pour comprendre, Des ailes sur un tracteur, Paris, 2014

Arnaud Alessandrin, Karine Espineira et Thomas Maud-Yeuse, «Transidentités : histoire d'une dépathologisation », Les Cahiers de la transidentité, vol. 1, 2013

Arnaud Alessandrin, «Le transsexualisme: une nosographie obsolète », Santé publique, 2012, p. 263-269

Arnaud Alessandrin, «La question Cisgenre », dans revue ¿ Interrogations ?, $\mathrm{N}^{\circ} 15$. Identité fictive et fictionnalisation de l'identité (I), décembre 2012 [en ligne]

Emmanuel Beaubatie, Alain Giami et Jonas Le Bail, «Caractéristiques sociodémographiques, identifications de genre, parcours médico-psychologique et VIH/sida dans la population trans », BEH, $\mathrm{n}^{\circ} 42,2011, \mathrm{p} .433-437$

Harry Benjamin, «Transvestism and transsexualism », International Journal of sexology, $\mathrm{n}^{\circ} 7,1953$

Judith Butler, Trouble dans le genre, La Découverte, Paris, 2005

Pierre-Henri Castel, La Métamorphose impensable, Gallimard, Paris, 2003

Colette Chiland et Bernard Cordier, «Transsexualisme», Encyclopédie médicochirurgicale, Elsevier, Paris, 2000

Agnes Condat et al., «Non-binarité et transidentités à l'adolescence », Neuropsychiatrie de l'enfance et de l'adolescence, vol. 66, $\mathrm{n}^{\circ}$ 6, 2018

Karine Espineira, Maud-Yeuse Thomas, Arnaud Alessandrin (dir.), « Tableau noir : les transidentités et l'école », Cahiers de la transidentité vol.4, 2014. 
Norman Fisk, «Gender dysphoria syndrome : the conceptualization that liberalizes indications for total gender reorientation and implies a broadly based multidimensional rehabilitative regimen » West Journal Med, n 120, 1974, p. 386-391

Harold Garkinkel, Recherches en ethnométhodologie, Presses universitaires de France, Paris, 2007

Gabrielle Richard, «Comment éduquer des enfants cisgenres ?», Actualité des trans studies, 2019, p. 31-38

Julia Serano, Whipping Girl, Seal Press, New York, 2007 